\title{
A Novel Sky Region Detection Algorithm Based On Border Points
}

\author{
Zhao Zhijie $^{1}$, Wu Qian ${ }^{1}$, Sun Huadong ${ }^{1}$, Jin Xuesong ${ }^{1,}$ Tian Qin $^{1}$ and Sun Xiaoying ${ }^{1}$ \\ 1. School of Computer and Information Engineering, Harbin University of \\ Commerce, Harbin 150028, China \\ zhijiezzz@aliyun.com,wuqian_kris@126.com
}

\begin{abstract}
Sky detection is an important problem in computer vision. Generally, the color and gradient of the pixel in sky border change greatly. So, sky in the scene can be detected by measuring the sky border points. In this paper, a new method for sky detection based on border points is proposed, where the border points in each column of image can be detected to complete complex sky region identification in image. Different to the existing sky detection methods, the proposed algorithm is efficient in detecting sky in scenes of complex structure, for example, sky which are separated by buildings or flags. Furthermore, the paper introduced the concept of sky border for the sky detection, which made the proposed scheme effective. The proposed method is able to handle even more complex sky conditions, including a cloudy sky and a sunny sky. Experimental results have proven that the effectiveness of this method.
\end{abstract}

Keywords: sky detection, border points, border position function, energy function

\section{Introduction}

The sky region detection plays a crucial role in many areas. In the application of the ground robots autonomous navigation [1], a robot perceives and understands the surrounding environment by the visual sensors. As sky is an important part of outdoor environment, a precise detection of sky regions provides the necessary environmental information for the robot [2]. As the sky is one of the most obvious features of the outdoor scenes, the sky detection can be used for scene classification, such as outdoor scene versus indoor scene, and city versus landscape. In the process of image retrieval based on spatial layout and location of sky region, sky detection is often employed to achieve more effective content-based image retrieval. In the process of detecting main subjects in the image, sky regions can usually be excluded because they are likely to be part of the background. In addition to the abovementioned applications, sky detection can be also used to improve image quality, depth map synthesis and so on. Generally, sky detection is important for computer vision.

Aditya Vailaya [3] proposed a sky detection method in 2000. In the proposed method, the colour and texture information was used as a combination. The image was firstly divided into blocks, and then colour and texture features were extracted from these blocks. Finally, a Bayesian classifier was employed to obtain correct sky regions. Gallagher, AC et al pointed out that a reasonable sky detection benefited image retrieval and understanding, and a sky detection method using two-dimensional polynomial model of images was proposed in 2004[4]. In that method, high confidence sky was firstly established, then two-dimensional polynomial model was used for candidate sky region to determine the final sky. However, the accuracy of detection was not high enough. In 2005, McGee T. proposed a sky detection method applied in obstacle detection system based on vision. Image was firstly converted into 
YCrCb colour space, and support vector machine was utilized to classify and obtain sky regions [5]. A sky detection method for the application of video image enhancement was presented by Zafarifar B. in 2007.They analysed the image and established an appropriate position colour model, a soft segmentation method was used to distinguish sky areas. But the method was dependent on spatially adaptive color model, therefore, its complexity was too high [6]. Laungrugthip et al. proposed a sky detection method for solar exposure prediction in 2008. For images which sunlight reflects off very shiny surfaces, the proposed method required some user interaction to identify the boundaries of the sky area [7]. Rankin A analysed the proportion of the image saturation and brightness, color intensity and edge gradient, and proposed a rule-based sky region detection method in 2011[8].

Most of above proposed methods adopt mathematical model or machine learning algorithm to detect sky. In addition, Ettinger S. proposed a vertical detection method in 2002. In this method, the sky and non-sky area was separated by a straight line which is determined by calculating the maximum energy function value. It is impossible to obtain accurate sky regions by only a simple line [9]. In 2013, Yehu Shen [10] improved the energy function and proposed a sky detection method based on Ettinger S's method. The proposed method could sketch out the shape of the boundary rather than use a straight line to separate the sky and non-sky region.

But in most urban landscape and photography pictures, the sky and the non-sky are not only separated by a boundary line. For example, there are buildings existing in the scenes, as shown in the left image of Figure 1. All of the sky region can not be obtained by only one border line. The right image of Fig. 1 is the result of the method in ref[10], it's known that this method can not detect the sky beneath the building. With that in mind, a new method is presented, where the concept of border point is proposed and border points in each column of image can be detected to complete complex sky identification.

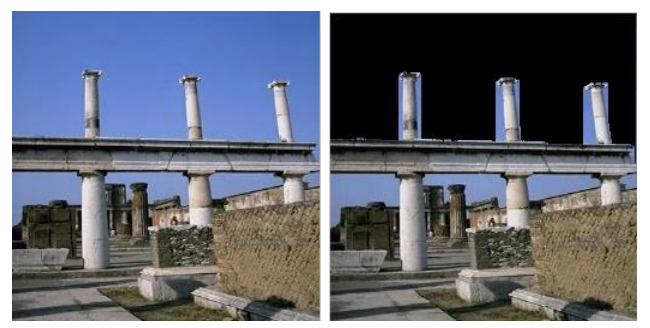

\section{Figure 1. Complex Sky Due to the Existing Buildings and Experimental Results of Method in Ref [10]}

\section{Background}

Reference [10] employed the image gradient information for sky detection, and the obtained maximum value of energy function from different sky border position functions was used to complete sky detection.

The definition of sky border position function is shown in equation (1):

$$
1 \leq b(x) \leq H(1 \leq x \leq W)
$$

where $\mathrm{W}$ and $\mathrm{H}$ are the width and height of the image, and $\mathrm{b}(\mathrm{x})$ determines the sky border position in the xth column. Thus the description of the sky and the non-sky region in image is shown as equation (2): 


$$
\begin{aligned}
& \text { sky }=\{(x, y) \mid 1 \leq x \leq W, 1 \leq y \leq b(x)\} \\
& \text { ground }=\{(x, y) \mid 1 \leq x \leq W, b(x)<y \leq H\}
\end{aligned}
$$

The following pseudo-codes show the algorithm for calculating the sky border position function $b(x)$.

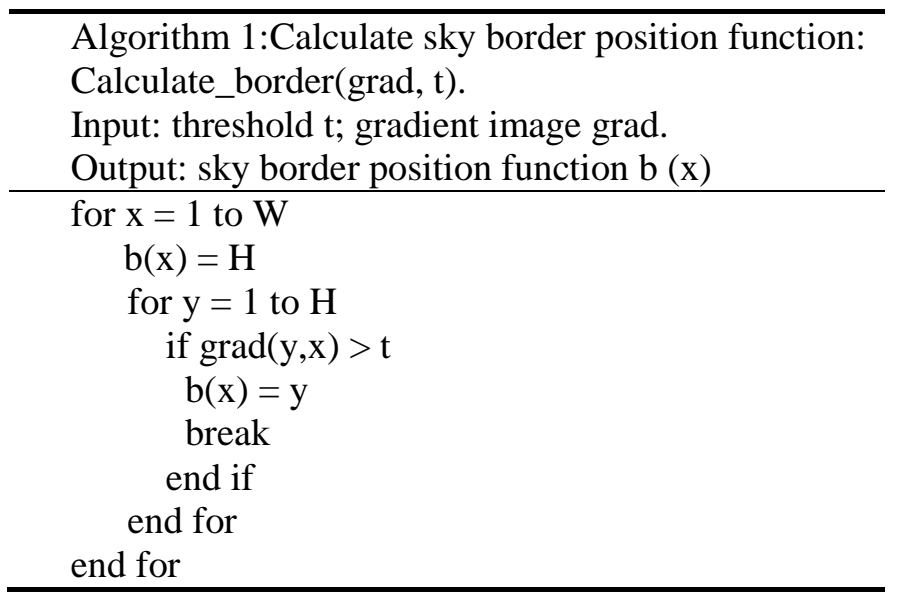

Then different sky border position functions can be obtained by custom threshold $\mathrm{t}$. Different values of energy function are calculated, sky region detection is completed by energy function optimization. Energy function is defined as equation (3):

$$
J_{n}=\frac{1}{\gamma\left|\sum_{s}\right|+\left|\sum_{g}\right|+\gamma\left|\lambda_{1}^{s}\right|+\left|\lambda_{1}^{g}\right|}
$$

$\Sigma_{s} \Sigma_{g}$ are the covariance matrix of pixels of sky and non-sky region, the region obtained by different sky border position functions, $\lambda_{1}^{s} \lambda_{1}^{g}$ are the first characteristic value of $\Sigma_{s} \Sigma_{g}$ respectively. If threshold t exceeds $600, \mathrm{Jn}(\mathrm{t})$ is nearly a constant. So the threshold and step is determined as follows:

$$
\text { thresh_min }=5 \text {, thresh_max }=600 \text {, search_step }=5
$$

The method in [10] added some post-processing for sky detection of some special scenes based on the above process, for example, scenes without a sky region and scenes columns without a sky region. Since this part of the discussion is not the focus of the method in this paper, it is not discussed here.

\section{Sky Detection Method based on Border Point}

\subsection{Definition of Border Point in Image}

The experimental image database comes from Corel image database [11] The Washington University School of Computer Science and Engineering [12]. Through statistics and analysis of a large number of images, we find that color and gradient of the pixels in sky border change greatly. As the sky region in image is smooth, gradient of pixels in this region is small. However, in the border of sky and non-sky, the gradient changes greatly.

Since the gradient also changes greatly when edges of objects exists and texture pattern changes a lot in the image. In contrast, sky region is smooth and it has some colour properties, which will be discussed later in this paper. The pixel which separates sky and nonsky region in each column of image is called border point. Red lines are the collection of the border points, as shown in figure 2. With the definition of border point, the entire sky region can be identified by various means. 


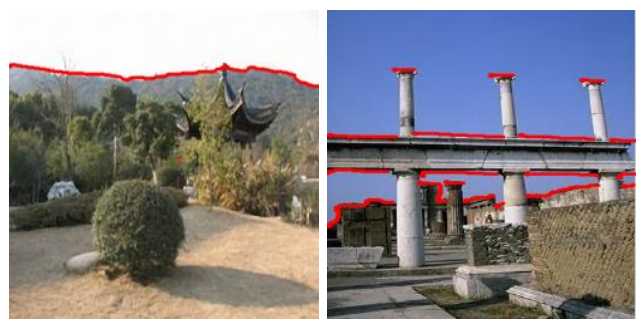

Figure 2. Red Lines are Collection of Border Points

As the method in ref [10] can only detect the first border point of each column in the image, if the complex sky border condition is appeared as shown in Fig.1, all of sky regions can not be detected by the above mentioned method. So a novel method for sky detection based on the method in ref [10] is presented. Colour feature and gradient feature of known sky region is utilized to determine border point and modify the sky border position function, so complex sky regions can be detected.

\subsection{Determination of Border Point}

The modifications of sky border position function in original method, namely the determination of multi-border points in presented in follows. Firstly, The images which have complex sky is pre-processed with the method in ref [10] and part sky region can be obtained, as shown the black region in Figure 1.

Since most of sky region is smooth, in other words, the gradient of sky is small or is close to zero; however, the gradient of border point is large. The first border point (sky area to non-sky area), is determined by a predefined threshold $t$ with gradient information of the image. The predefined threshold $t$ is shown as equation (4).

The second border point (non-sky region to sky region) is determined by statistics of sky colour information, the sky which is obtained through pre-treatment. It's given that most sky regions are blue, namely that $\mathrm{b}$ component of this region is great. Therefore, $\mathrm{b}$ component of color is considered to determine the second border point. But white objects are often appeared in the scene, for example, white buildings and white cars. They have parallel characteristics in the ways of gradient and b component compared with sky region. Therefore, in order to detect sky regions correctly, the judgment of $r$ and g components should be added. Through a lot of statistics of sky obtained through pre-treatment, the average of each color component can reflect characteristics of sky region in some extent. So, average of color component is as judgment and different input image can adjust adaptively. The specific form is shown as follows. The pixel to be detected satisfying equation (5) is the second border point:

$$
r_{x}-r_{\text {arg }}<\delta_{1} a n d g_{x}-g_{\text {arg }}<\delta_{2} a n d b_{\text {arg }}-b_{x}<\delta_{3}
$$

$r_{\text {arg }} g_{\text {arg }} b_{\text {arg }}$ are the average value of $\mathrm{r}$, g and $\mathrm{b}$ components of known sky region, $r_{x} g_{x} b_{x}$ denote $\mathrm{r}, \mathrm{g}$ and $\mathrm{b}$ components of the pixel to be detected. $\delta_{1} \delta_{2} \delta_{3}$ represent the floating range. $\delta_{1} \delta_{2} \delta_{3}$ equals to ten percent of $r_{\text {arg }} g_{\text {arg }} b_{\text {arg }}$ respectively. They are empirical parameters which are derived through a number of experiments.

The third border point is similar to the first border point, so it is determined as the first border point.

It is noted that if the second border point of one column is not found, then keeping on finding border point in the next column. It is indicated that there is no sky separated in this column of image. 
Sky border position function can be modified after determination of each border point, and sky and non-sky regions corresponding to different border position functions can be obtained. If there is only one border point in the column, sky border position function of this column is shown as equation (1), and corresponding sky and non-sky region in this column is defined in equation (2). If more than one border points are detected in the column, the sky border position function and the corresponding sky and non-sky region are defined as equation (6):

$$
\begin{aligned}
& \quad 1 \leq b(x)_{1}, b(x)_{2}, b(x)_{3} \leq H(1 \leq \mathrm{x} \leq W) \\
& \text { sky }=\left\{(x, y) \mid 1 \leq x \leq W,\left(1 \leq y \leq b(x)_{1}\right) \text { and }\left(b(x)_{2} \leq y \leq b(x)_{3}\right)\right\} \\
& \text { ground }=\left\{(x, y) \mid 1 \leq x \leq W,\left(b(x)_{1}<y<b(x)_{2}\right) \text { and }\left(b(x)_{3}<y \leq H\right)\right\}
\end{aligned}
$$

The input image is pre-processed, and then the determination of border points of each column is shown as flow chart in Figure 3:

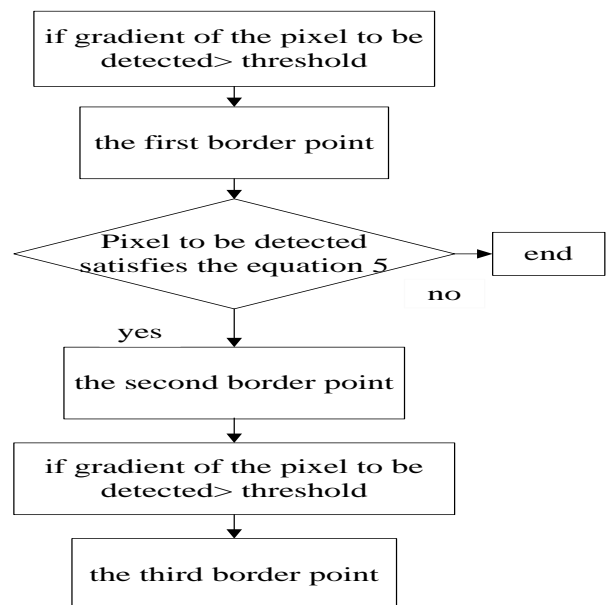

Figure 3. The Flow Chart of Calculation of Sky Border Position Function

\subsection{Outline of Proposed Method}

(1)The input image is pre-processed with the method in ref [10], and then color information of obtained sky region is counted.

(2)The input image is converted into a greyscale image, its corresponding gradient image with the sobel operator is calculated [13].

(3)Sky border points of each column are determined, and sky and non-sky areas are obtained by equation (1), (2) and (6).

(4)Energy function value of sky and non-sky region is calculated by equation (3).

(5)The threshold is changed, and step 3 and 4 is repeated.

(6)Entire sky regions are detected by searching sky and non-sky region corresponding to maximum energy function value.

\section{Experimental Results and Analysis}

The method in ref [10] is improved, this paper is more concentrated about the detection of sky regions which are separated by buildings or flags. The proposed method can also handle even more complex sky conditions, including a cloudy sky and a sunny sky. Border points can be detected in each column of input image by the proposed algorithm, and then all sky region can be obtained. Experimental results show 
that the proposed method can efficiently detect sky regions of image, and it also reduces the computational complexity rather than use mathematics models and machine learning algorithm.

In order to verify the effectiveness of the proposed method in this paper, namely the accuracy of the determination of multi-border points, the following common types of pictures are selected. The experimental image database comes from Corel image database [11] The Washington University School of Computer Science and Engineering [12].

Due to the smooth properties of sky, so gradient information of the input image is only considered in the method of this paper initially, namely the second border point is only determined with gradient information, however, the result is not ideal. For this reason, judgment of b component is added, since sky is blue in most of pictures. Experimental results show that the initial method is applicable for most images, but for the white areas which are in the buildings or flags, it lead to the error detection, as shown in Figure 4, the black part is detected as sky region. The first image in left column of Figure 4 is input image, due to the white pattern in the flag, the method lead to error detection of second border point, so the real sky regions are not detected correctly. There is no separate sky region in second input image of left column, but owing to the existence of white small car in image, it is mistaken as the sky region.

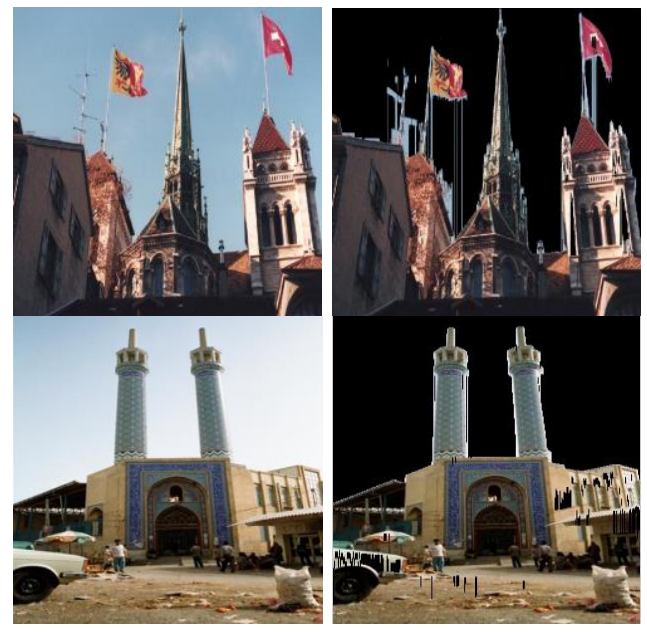

Figure 4. The Left Column are Input Images, the Right Column are the Experimental Results which the Second Border Point is only determined by Gradient Information and b Component of Color

The $\mathrm{r}, \mathrm{g}, \mathrm{b}$ components of pixels in white areas of image are great, therefore, $\mathrm{r}$ and $\mathrm{g}$ components were considered to be added in the judgment of the second border point, detail form is shown in equation (5).The results with gradient and color information are shown as Figure 5. 


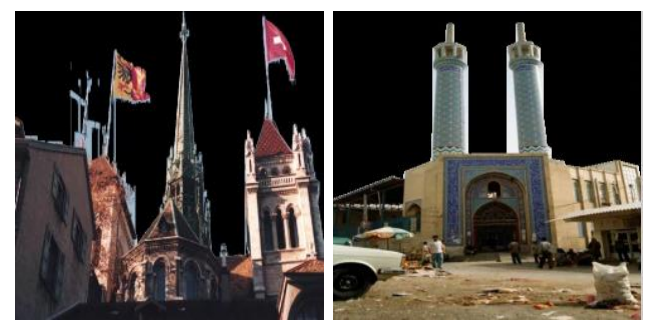

\section{Figure 5. The Detection Results which the Second Border Point is Determined by Gradient and Color Information}

Since the gradient image is obtained by the gray image with Sobel operator, and the gray image is converted from color image, therefore color information of image should contain gradient information. Experimental results prove our assumptions, which the second border point is determined only by color information. The result with gradient and color information is as same as the result with only color information. Therefore, the flow of border point's determination is shown as Figure 3.

It's known that the proposed method is feasible and effective compared with the method in ref [10]. The results are shown in Figure 6, where the black regions are detected sky regions.
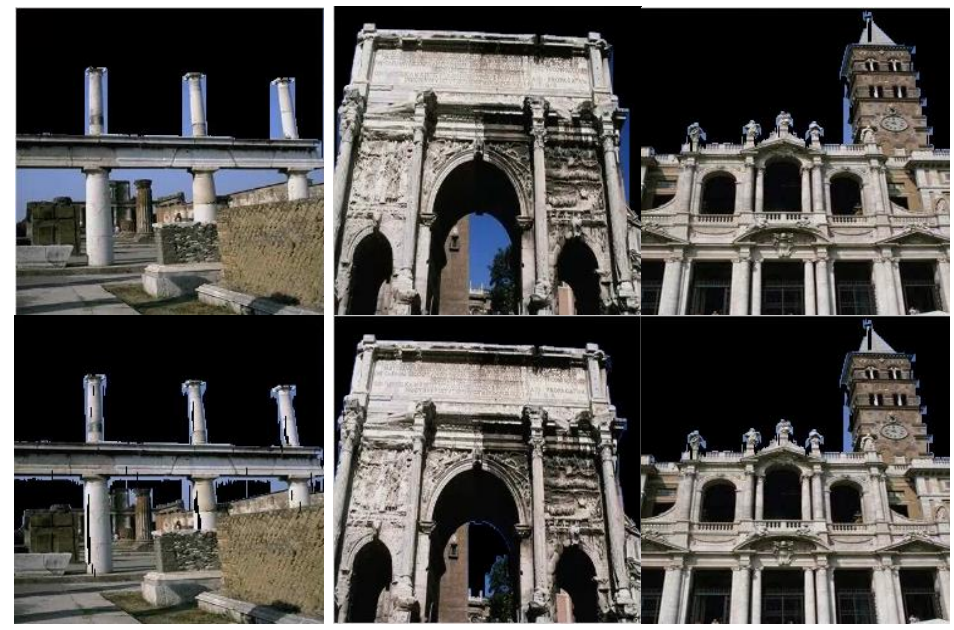

Figure 6. The First Row are the Results of the Method in Ref [10], the Second Row are the Results of the Proposed Method

The sky detection method proposed in this paper is applied to common complex images. However, the results are not ideal for more complex sky cases. As shown in Figure 7, due to complex ferris wheel and petals patterns in image, some sky regions are not detected. As there are more than three border points in the columns in these images. For this type of pictures, other characteristics of the sky region will be considered in the future work, entire sky regions will be detected by matching or searching methods and so on. 

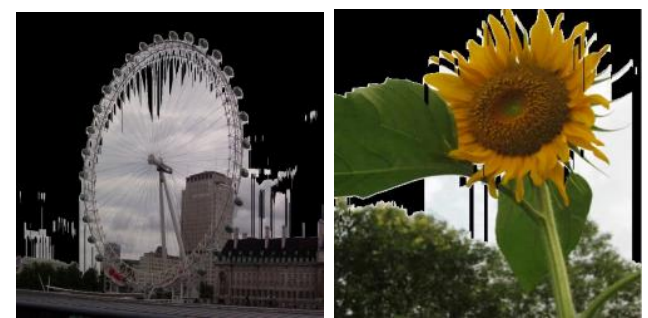

Figure 7. The Detection Result of Complex Sky by Method based on Border Points

\section{Conclusions}

In this paper, a novel sky region detection algorithm is presented, and the border points in each column of image can be detected to complete complex sky region detection in image. The proposed method introduces a new concept of sky border for the sky detection, which made the proposed scheme simple but effective. The proposed method is feasible and effective for the images which sky regions are separated by complex objects such as flags or buildings. The further work is to improve the accuracy of sky detection with other image information.

\section{Acknowledgements}

This work was supported by Scientific Research Foundation for Returned Scholars, Ministry of Human Resources and Social Security, Natural Science Foundation of Heilongjiang Province (F201245) and technological innovation foundation of Harbin (2014RFQXJ166)

\section{References}

[1] N. Kim., H. H. Trinh and K. H. Jo, "Regions Segmentation Using Multiple Cues for Robot Navigation on Outdoor Environment, International Conference on Control, Automation and Systems,(2007), Seoul. Korea.

[2] S. Thrun and M Mike, "Stanley: The Robot that Won the DARPA Grand Challenge", Journal of Field Robotics, vol. 23, no. 9, (2006).

[3] A. Vailaya and A. K. Jain, "Detecting Sky and Vegetation in Outdoor Image", Conference on Storage and Retrieval for Media Databases, (2000), pp. 411-420.

[4] A. C. Gallagher, J. Luo, and H. Wei, "Improved Blue Sky Detection Using Polynomial Model Fit", International Conference on Image Processing, (2004), p. 2367 - 2370.

[5] T. G. McGee, R. Senqupta and K. Hedrick, "Obstacle Detection for Small Autonomous Aircraft Using Sky Segmentation", International Conference on Robotics and Automation, (2005), pp. 4679-4684.

[6] B. Zafarifar and H. N. Peter, "Blue Sky Detection for Content-based Television Picture Quality Enhancement”, International Conference on Consumer Electronics, Las Vegas, US, (2007), p. 1-2.

[7] N. Laungrungthip, A. E. McKinnon., and C. D. Churcher,'Edge-Based Detection of Sky Regions in Images for Solar Exposure Prediction", 23rd International Conference on Image and Vision Computing New Zealand, Christchurch, New Zealand, (2008), p. 1-6.

[8] A. Rankin, L. Matthies and P. Bellutta, "Daytime Water Detection Based on Sky Reflections", International Conference on Robotics and Automation, Shanghai, China, (2011), p. 5329-5336.

[9] S. M. Ettinger., C. N. Michael, G. I. Peter and W. Martin, "Vision-Guided Flight Stability and Control for Micro Air Vehicles", International Conference on Intelligent Robots and Systems, (2002), p. 2134-2140.

[10] Y. H. Shen and Q. C. Wang, "Sky Region Detection in a Single Image for Autonomous Ground Robot Navigation", International Journal of Advanced Robotic Systems, vol. 10, (2013).

[11] http://wang.ist.psu.edu/docs/related/

[12] http://www.cs.washington.edu/research/imagedatabase

[13] R. Gonzales and R. Woods, "Digital Image Processing”, Upper Saddle River: Prentice-Hall Press (2008). 


\section{Authors}

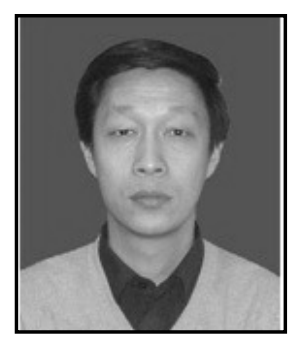

Zhijie Zhao, Doctor, Professor, IEEE Member, His research fields are image processing and intelligence information processing.

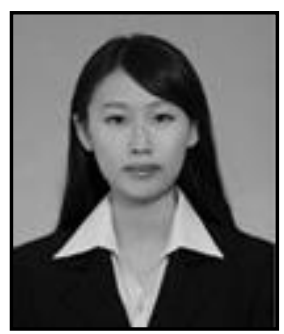

Qian Wu, Harbin University of Commerce post-graduation, Her research fields are computer networks and communications 
International Journal of Signal Processing, Image Processing and Pattern Recognition Vol.8, No.3 (2015) 